\title{
Cubic finite state machine and cubic transformation semigroups
}

\author{
Saleem Abdullah, Rabia Naz and Witold Pedrycz \\ Department of Mathematics, Quaid-i-Azam University Islamabad Pakistan \\ Department of Mathematics, FC College, Lahor, Pakistan \\ Department of Computer Engineering, University of Alberta, Canada
}

Received: 3 January 2016, Accepted: 7 December 2016

Published online: 20 October 2017.

\begin{abstract}
This paper provides a new generalization of fuzzy finite state machines, fuzzy transformation semigroups and their relationship. Consider a cubic structure, we introduce cubic finite state machines, cubic transformation semigroups, cubic successor, cubic exchange properties cubic subsystems, cubic submachines, cubic q-twins, cubic retrievable and study fundamental properties of them. We provide relationship between cubic $q$-twins and a cubic $q$-related. We provide a characterization of a cubic retrievable. We define cfsm homomorphism and investigated related properties. We show that the composition of strong cfsm homomorphism is also strong. We also define cubic transformation semigroup and it related properties. We define cts homomorphism and its properties.
\end{abstract}

Keywords: Cubic finite state machine, cubic transformation semigroups.

\section{Introduction}

Mathematical models of classical computation, automata have been an important area in theoretical computer science [37]. It started from a seminal paper of Kleene [38], and within a few years developed into a rich mathematical research topic. From the very beginning finite automata constituted a core of computer science. One of the reason is that they capture very fundamental ideas is witnessed by a numerous different characterizations of the family of rational languages, i.e. languages defined by finite automata [37]. In fact, the interrelation of finite automata and their applications in computer science is a splendid example of a really fruitful connection of theory, practice and these will accept regular language [39]. Finite automata played a crucial role in the theory of programming languages, compiler constructions, switching circuit design, controllers, neural networks, text editor and lexical analyzer [41]. The topic of these investigations belongs to the theoretical soft computing (fuzzy structures). Indeed, it is well known that semigroups are basic structures in many applied branches of information sciences, like finite state machines or automata, formal languages, coding theory and others. To be more precise, structure theory of finite state machines (automata) is based on transformation semigroups. Algebraic automata theory provide powerful decomposition results for finite transformation semigroups by cascade simple parts. Any full transformation semigroup is regular. The fuzzy set theory, proposed by Zadeh [42] is a framework for dealing uncertainty. The literature on fuzzy set theory and its applications have been growing rapidly. Research on fuzzy set theory has received much attention in recent years. Applications of fuzzy set theory have been studied in different areas, that is, artificial intelligence, computer sciences, control engineering, expert, robotics, automat theory, finite state machine, graph theory and others. Fuzzy set is a type of important mathematical structure to epitomize a assortment of objects whose boundary is vague. There are some type of fuzzy set extensions in the fuzzy set theory, for example: interval-valued fuzzy sets, intuitionistic fuzzy sets, bipolar fuzzy set and vague sets etc. Cubic set which was introduced by Jun [36], is an extension of fuzzy set and interval valued fuzzy set and intuitionistic 
fuzzy set. Jun combined interval valued fuzzy set and fuzzy set to introduced cubic sets and also defined P-union, P-intersection, R-union and R-intersection of cubic sets, and investigate several related properties. Cubic sets is a new framework for dealing uncertainty when fuzzy sets, interval valued fuzzy sets and intuitionistic fuzzy sets fail dealing uncertainty.

The concept of transformation semigroup, covering, cascade product and wreath product play vital role in the study of automata [23]. Since Wee [20] in 1967 introduced the concept of fuzzy automata following Zadeh [42], fuzzy automata theory has been developed by many researchers. Malik et al. [2,3,4,5] introduced the concepts of fuzzy state machines and fuzzy transformation semigroups based on Wee's concept of fuzzy automata and related concepts and applied algebraic technique. In [1], Kim et al. introduced the notion of T-generalized state machine and T-generalized transformation semigroup that are extensions of fuzzy state machine and fuzzy transformation semigroup, respectively. They defined the notions of fuzzy finite state submachine of a fuzzy finite state machine, retrievable, separated and connected fuzzy finite state machines and discussed their basic properties. They also introduced a decomposition theorem for fuzzy finite state machines in terms of primary fuzzy finite state submachines. Kumbhojkar and Chaudhari [25] gave different ways of constructing products of fuzzy finite state machines and their mutual relationship, through isomorphism and coverings.

Algebraic technique is very significant in the study of fuzzy automat theory [2,3,4,5]. Algebraic properties and minimization of fuzzy automata and fuzzy languages studied by many authors $[7,8,9,10,11,12,13,14,15]$. One of the essential problems in algebraic fuzzy automata theory is decomposition theory of fuzzy automata, which gives a way for stimulating complex machine by simpler machine [23]. In classical fuzzy automata, the decomposition theorems have been given by utilizing successor operators and submachines [34,35]. In [33], Bavel introduced source and successor operators to used explore subautomata, separability, retrievability and homomorphism. Moreover, these operators are often applied to topology, fuzzy directed graphs, fuzzy abstract algebra, model logic and interval structure [26,27,28, 40]. Recently, Jun have been introduced intuitionistic ffsms and intuitionistic ftss, as well as studied their relationship (see $[16,17,18,19])$, but it is clear, looking through the literature, that the cubic sets are more advisable in order to model uncertainty in some applications. Taking cubic structure, We defined cubic finite state machine (cfsm) and cubic transformation semigroups (cts) as a generalization of fuzzy finite state machine, fuzzy transformation semigroups [2,3, $4,5]$, intuitionistic fuzzy finite state machine and intuitionistic fuzzy transformation semigroups $[16,17,18,19]$. We also define cubic successor, cubic exchange properties cubic subsystems, cubic submachines, cubic q-twins, cubic retrievable, and study related properties. Using a cubic finite state machine, we make two finite semigroups with identity, and show that they are isomorphic. We also introduce the notion of cubic admissible relation, and initiate its characterization. We establish an isomorphism between a cubic finite state machine and the quotient structure of another cubic finite state machine.

In this article, we apply the notion of cubic sets to finite state machine to introduce the concept of cubic finite state machines, cubic transformation semigroups, cubic successor, cubic exchange properties cubic subsystems, cubic submachines, cubic q-twins, cubic retrievable, and study related properties. We show relationship between cubic $q$-twins and a cubic $q$-related. We provide a characterization of a cubic retrievable. We define cfsm homomorphism and investigated related properties. We show that the composition of strong cfsm homomorphism is also strong. We also define cubic transformation semigroup and it related properties. We define cts homomorphism and its properties.

\section{Basic concept}

A map $\lambda: X \rightarrow[0,1]$ is called a fuzzy set of $X$. A function $A: X \rightarrow[I]$ is called an interval-valued fuzzy set (shortly, an IVF set) in $X$. Let $[I]$ stands for the set of all sub interval of $[0,1]$ and $[I]^{X}$ stand for the set of all IVF of $X$. For every 
$A \in[I]^{X}$ and $x \in X, A(x)=\left[A^{-}(x), A^{+}(x)\right]$ is called the degree of membership of an element $x$ to $A$, where $A^{-}: X \rightarrow I$ and $A^{+}: X \rightarrow I$ are fuzzy sets in $X$ which are called a lower fuzzy set and an upper fuzzy set in $X$, respectively. For simplicity, we denote $A=\left[A^{-}, A^{+}\right]$. For every $A, B \in[I]^{X}$, we define $A \subseteq B$ if and only if $A(x) \preceq B(x)$ for all $x \in X$. A map $\mathscr{A}=\langle\widetilde{A}, \lambda\rangle: X \rightarrow[I] \times[0,1]$ is called a cubic set.

Definition 1. A fuzzy finite state machine (fsm, for short) is a triple $\mathscr{M}=\langle Q, X, \delta\rangle$ where $Q$ and $X$ are finite non-empty sets, called the set of states and the set of input symbols, respectively, and $\delta$ is a fuzzy set in $Q \times X \times Q$.

Definition 2. Let $\boldsymbol{\aleph}=\langle Q, M, \delta\rangle$ be a ffsm. Then, define a fuzzy set $\delta^{*}$ in $Q \times M^{*} \times Q$ as follow:

$$
\begin{aligned}
\delta^{*}(q, \varepsilon, p) & =\left\{\begin{array}{l}
1 \text { if } q=p \\
0 \text { if } q \neq p
\end{array}\right. \\
\delta^{*}(q, m n, p) & =\bigvee_{r \in Q}\left\{\delta^{*}(q, m, r) \wedge \delta^{*}(r, n, p)\right\}
\end{aligned}
$$

for all $p, q \in Q, m, a \in M^{*}$.

Definition 3. A fuzzy transformation semigroups (in short, fts) is a triple $\mathscr{S}=\langle Q, X, \lambda\rangle$, where $Q$ is a non-empty finite set, $X$ is a finite semigroup with identity $\varepsilon$ and $\lambda$ is a fuzzy set in $Q \times X \times Q$ such that

$(T S 1)$

$$
\lambda(q, \varepsilon, p)=\left\{\begin{array}{l}
1 \text { if } q=p \\
0 \text { if } q \neq p
\end{array}\right.
$$

$$
\lambda(q, x y, p)=\bigvee_{r \in Q}\{\lambda(q, x, r) \wedge \lambda(r, y, p)\}
$$

for all $p, q \in Q, x, y \in X$.

\section{Cubic finite state machine}

In [2,5], Malik et al. defined fuzzy finite state machine (automata) based on Wee's concept of fuzzy automata and related concepts and applied algebraic technique. Jun introduced the concept of intuitionistic fuzzy finite state machine and studied fundamental results. We define cubic finite state machines, cubic successor, cubic exchange properties cubic subsystems, cubic submachines, cubic q-twins, cubic retrievable, and study related properties. The present concept is generalization the concept of Malik et al [2,5] and Jun concept [17].

Definition 4. A cubic finite state machine (cfsm, shortly) is a triple $\aleph=\langle Q, M, \mathscr{A}\rangle$, where $Q$ and $M$ are finite non-empty sets, called the set of states and the set of input symbols, respectively, and $\mathscr{A}=\langle\widetilde{A}, \lambda\rangle$ is a cubic set in $Q \times M \times Q$.

Example 1. Let $Q=\left\{p_{1}, p_{2}, p_{3}, p_{4}, p_{5}, p_{6}, p_{7}\right\}$ and $X=\{x\}$ and let $\mathscr{A}=\langle\widetilde{A}, \lambda\rangle$ be a cubic set in $Q \times M \times Q$ defined by

$$
\begin{aligned}
\widetilde{A}\left(p_{1}, x, p_{2}\right) & =[0.7,0.8], \widetilde{A}\left(p_{1}, x, p_{3}\right)=[0.3,0.35], \widetilde{A}\left(p_{1}, x, p_{n}\right)=\widetilde{0}, n=1,4, \ldots 7 \\
\widetilde{A}\left(p_{2}, x, p_{4}\right) & =[0.6,0.65], \widetilde{A}\left(p_{1}, x, p_{5}\right)=[0.4,0.45], \widetilde{A}\left(p_{1}, x, p_{m}\right)=\widetilde{0}, m=1,2,3,6,7 \\
\widetilde{A}\left(p_{3}, x, p_{6}\right) & =[0.1,0.15], \widetilde{A}\left(p_{1}, x, p_{7}\right)=[0.6,0.65], \widetilde{A}\left(p_{1}, x, p_{l}\right)=\widetilde{0}, l=1,2, \ldots 6 \\
\widetilde{A}\left(p_{s}, x, p_{t}\right) & =[0,0.2], s=4, \ldots 7 \text { and } t=1, \ldots 7
\end{aligned}
$$


and

$$
\begin{aligned}
& \tilde{\lambda}\left(p_{1}, x, p_{2}\right)=0.7, \lambda\left(p_{1}, x, p_{3}\right)=0.3, \lambda\left(p_{1}, x, p_{n}\right)=0, n=1,4, \ldots 7 \\
& \lambda\left(p_{2}, x, p_{4}\right)=0.6, \lambda\left(p_{1}, x, p_{5}\right)=0.4, \lambda\left(p_{1}, x, p_{m}\right)=0, m=1,2,3,6,7 \\
& \lambda\left(p_{3}, x, p_{6}\right)=0.1, \lambda\left(p_{1}, x, p_{7}\right)=0.6, \lambda\left(p_{1}, x, p_{l}\right)=0, l=1,2, \ldots 6 \\
& \lambda\left(p_{s}, x, p_{t}\right)=0.1, s=4, \ldots 7 \text { and } t=1, \ldots 7
\end{aligned}
$$

Thus, $\mathfrak{\aleph}=\langle Q, M, \mathscr{A}\rangle$ is a cubic finite state machine

Let $M^{*}$ denote the set of all words of elements of $M$ of finite length. Let $|m|$ denote the length of $m$ for every $m \in M^{*}$ and $\varepsilon$ denote the the empty word in $M^{*}$. We generalized the following definition by using the $\widetilde{t}$-norm and cot-norm.

Definition 5. Let $\mathbb{\aleph}=\langle Q, M, \mathscr{A}\rangle$ be a cfsm. Then, define a cubic set $\mathscr{A}^{*}=\left\langle\widetilde{A^{*}}, \lambda^{*}\right\rangle$ in $Q \times M^{*} \times Q$ as follow

$$
\widetilde{A^{*}}(q, \varepsilon, p)=\left\{\begin{array}{l}
\widetilde{1} \text { if } q=p \\
\widetilde{0} \text { if } q \neq p
\end{array} \quad \lambda^{*}(q, \varepsilon, p)=\left\{\begin{array}{l}
0 \text { if } q=p \\
1 \text { if } q \neq p
\end{array}\right.\right.
$$

and

$$
\begin{aligned}
& \widetilde{A^{*}}(q, m n, p)=\bigvee_{r \in Q}\left\{\widetilde{A^{*}}(q, m, r) \wedge \widetilde{A^{*}}(r, n, p)\right\} \\
& \lambda^{*}(q, m n, p)=\bigwedge_{r \in Q}\left\{\lambda^{*}(q, m, r) \vee \lambda^{*}(r, n, p)\right\}
\end{aligned}
$$

for all $p, q \in Q, m \in M^{*}$ and $n \in M$.

Proposition 1. Let $\aleph=\langle Q, M, \mathscr{A}\rangle$ be a cfsm. Then, the following hold

$$
\widetilde{A^{*}}(q, m a, p)=\bigvee_{r \in Q}\left\{\widetilde{A^{*}}(q, m, r) \wedge \widetilde{A^{*}}(r, a, p)\right\}
$$

and

$$
\lambda^{*}(q, m a, p)=\bigwedge_{r \in Q}\left\{\lambda^{*}(q, m, r) \vee \lambda^{*}(r, a, p)\right\}
$$

for all $p, q \in Q, m, a \in M^{*}$.

Proof. Take $p, q \in Q$ and $m, n \in M^{*}$. Then, we shall prove the result by using induction on $|a|=n$. In case of $n=0$, then $a=\varepsilon$ and so $m a=m \varepsilon=m$. Hence,

$$
\begin{aligned}
\bigvee_{r \in Q}\left\{\widetilde{A^{*}}(q, m, r) \wedge \widetilde{A^{*}}(r, a, p)\right\} & =\bigvee_{r \in Q}\left\{\widetilde{A^{*}}(q, m, r) \wedge \widetilde{A^{*}}(r, \varepsilon, p)\right\} \\
& =\widetilde{A^{*}}(q, m \varepsilon, r)=\widetilde{A^{*}}(q, m a, r) \\
\widetilde{A^{*}}(q, m a, r) & =\bigvee_{r \in Q}\left\{\widetilde{A^{*}}(q, m, r) \wedge \widetilde{A^{*}}(r, a, p)\right\}
\end{aligned}
$$


and

$$
\begin{aligned}
\bigwedge_{r \in Q}\left\{\lambda^{*}(q, m, r) \wedge \lambda^{*}(r, a, p)\right\} & =\bigwedge_{r \in Q}\left\{\lambda^{*}(q, m, r) \vee \lambda^{*}(r, \varepsilon, p)\right\} \\
& =\lambda^{*}(q, m \varepsilon, r)=\lambda^{*}(q, m a, r) \\
\lambda^{*}(q, m a, r) & =\bigwedge_{r \in Q}\left\{\lambda^{*}(q, m, r) \vee \lambda^{*}(r, a, p)\right\} .
\end{aligned}
$$

Hence, the result is valid for $n=0$. Assume that the result is true for all $u \in M^{*}$ such that $|u|=n-1, n>0$. Let $a=u v$, where $u \in M^{*}$ and $v \in M$. Then,

$$
\begin{aligned}
\widetilde{A^{*}}(q, m a, r) & =\widetilde{A^{*}}(q, m u v, r)=\bigvee_{r \in Q}\left\{\widetilde{A^{*}}(q, m u, r) \wedge \widetilde{A^{*}}(r, v, p)\right\} \\
& =\bigvee_{r \in Q}\left\{\bigvee_{s \in Q}\left\{\widetilde{A^{*}}(q, m, s) \wedge \widetilde{A^{*}}(s, u, r)\right\} \wedge \widetilde{A^{*}}(r, v, p)\right\} \\
& =\bigvee_{r, s \in Q}\left\{\widetilde{A^{*}}(q, m, s) \wedge \widetilde{A^{*}}(s, u, r) \wedge \widetilde{A^{*}}(r, v, p)\right\} \\
& =\bigvee_{s \in Q}\left\{\widetilde{A^{*}}(q, m, s) \wedge\left(\bigvee_{r \in Q}\left\{\widetilde{A^{*}}(s, u, r) \wedge \widetilde{A^{*}}(r, v, p)\right\}\right)\right\} \\
& =\bigvee_{s \in Q}\left\{\widetilde{A^{*}}(q, m, s) \wedge \widetilde{A^{*}}(s, u v, p)\right\} \\
& =\bigvee_{s \in Q}\left\{\widetilde{A^{*}}(q, m, s) \wedge \widetilde{A^{*}}(s, a, p)\right\}
\end{aligned}
$$

and

$$
\begin{aligned}
\lambda^{*}(q, m a, r) & =\lambda^{*}(q, m u v, r)=\bigwedge_{r \in Q}\left\{\lambda^{*}(q, m u, r) \vee \lambda^{*}(r, v, p)\right\} \\
& =\bigwedge_{r \in Q}\left\{\bigwedge_{s \in Q}\left\{\lambda^{*}(q, m, s) \vee \lambda^{*}(s, u, r)\right\} \vee \lambda^{*}(r, v, p)\right\} \\
& =\bigwedge_{r, s \in Q}\left\{\lambda^{*}(q, m, s) \vee \lambda^{*}(s, u, r) \vee \lambda^{*}(r, v, p)\right\} \\
& =\bigwedge_{s \in Q}\left\{\lambda^{*}(q, m, s) \vee\left(\bigwedge_{r \in Q}\left\{\lambda^{*}(s, u, r) \vee \lambda^{*}(r, v, p)\right\}\right)\right\} \\
& =\bigwedge_{s \in Q}\left\{\lambda^{*}(q, m, s) \vee \lambda^{*}(s, u v, p)\right\} \\
& =\bigwedge_{s \in Q}\left\{\lambda^{*}(q, m, s) \vee \lambda^{*}(s, a, p)\right\} .
\end{aligned}
$$

Thus, the result is true for $|a|=n$.

Definition 6. Let $\aleph_{1}=\left\langle Q_{1}, M_{1}, \mathscr{A}_{1}\right\rangle$ and $\aleph_{2}=\left\langle Q_{2}, M_{2}, \mathscr{A}_{2}\right\rangle$ be two cfsms. A cfsm homomorphism from $\aleph_{1}$ to $\aleph_{2}$ is fair $(\xi, \eta)$ of mappings, $\xi: Q_{1} \longrightarrow Q_{2}$ and $\eta: M_{1} \longrightarrow M_{2}$, such that

$$
\widetilde{A_{1}}\left(q_{1}, m_{1}, p_{1}\right) \preceq \widetilde{A_{2}}\left(\xi\left(q_{1}\right), \eta\left(m_{1}\right), \xi\left(p_{1}\right)\right)
$$


and

$$
\lambda_{1}\left(q_{1}, m_{1}, p_{1}\right) \geq \lambda_{2}\left(\xi\left(q_{1}\right), \eta\left(m_{1}\right), \xi\left(p_{1}\right)\right) \forall p_{1}, q_{2} \in Q_{1} \text { and } m_{1} \in M_{1} .
$$

We denoted by $(\xi, \eta): \aleph_{1} \longrightarrow \aleph_{2}$.

A cfsm homomorphism $(\xi, \eta)$ is called strong if, in addition, for any $p_{1}, q_{1} \in Q_{1}$ and $m_{1} \in M_{1}$,

$$
\widetilde{A_{2}}\left(\xi\left(q_{1}\right), \eta\left(m_{1}\right), \xi\left(p_{1}\right)\right)=\bigvee\left\{\widetilde{A_{1}}\left(q_{1}, m_{1}, r_{1}\right) \mid \xi\left(r_{1}\right)=\xi\left(p_{1}\right)\right\}
$$

and

$$
\lambda_{2}\left(\xi\left(q_{1}\right), \eta\left(m_{1}\right), \xi\left(p_{1}\right)\right)=\bigwedge\left\{\lambda_{1}\left(q_{1}, m_{1}, r_{1}\right) \mid \eta\left(r_{1}\right)=\eta\left(p_{1}\right)\right\} .
$$

A cfsm homomorphism $(\xi, \eta)$ is called isomorphism if, both mappings $\xi$ and $\eta$ are bijective.

Remark. If $(\xi, \eta): \aleph_{1} \longrightarrow \aleph_{2}$ is a strong cfsm homomorphism and one-to-one, then

$$
\widetilde{A_{1}}\left(q_{1}, m_{1}, r_{1}\right)=\widetilde{A_{2}}\left(\left(\xi\left(q_{1}\right), \eta\left(m_{1}\right), \xi\left(p_{1}\right)\right)\right.
$$

and

$$
\lambda_{1}\left(q_{1}, m_{1}, r_{1}\right)=\lambda_{2}\left(\xi\left(q_{1}\right), \eta\left(m_{1}\right), \xi\left(p_{1}\right)\right), \forall p_{1}, q_{1} \in Q_{1} \text { and } m_{1} \in M_{1} .
$$

Theorem 1. Let $\left(\xi_{1}, \eta_{1}\right): \aleph_{1} \longrightarrow \aleph_{2}$ and $\left(\xi_{2}, \eta_{2}\right): \aleph_{2} \longrightarrow \aleph_{3}$ be strong cfsm homomorphism between the cfsms $\aleph_{1}=$ $\left\langle Q_{1}, M_{1}, \mathscr{A}_{1}\right\rangle, \aleph_{1}=\left\langle Q_{2}, M_{2}, \mathscr{A}_{2}\right\rangle$ and $\aleph_{3}=\left\langle Q_{3}, M_{3}, \mathscr{A}_{3}\right\rangle$.

(1) Let $\xi_{1}$ be onto. Then, the composition $\left(\xi_{2}, \eta_{2}\right) \circ\left(\xi_{1}, \eta_{1}\right): \aleph_{1} \longrightarrow \aleph_{3}$ is a strong cfsm homomorphism.

(2) Let $\xi_{1}$ and $\xi_{2}$ be one-to-one. Then, the composed cfsm homomorphism is also strong.

Proof. (1) Since $\left(\xi_{1}, \eta_{1}\right): \aleph_{1} \longrightarrow \aleph_{2}$ and $\left(\xi_{2}, \eta_{2}\right): \aleph_{2} \longrightarrow \aleph_{3}$ are both strong homomorphism, for any $p_{1}, q_{1} \in Q_{1}$ and $m_{1} \in M_{1}$, we have

$$
\begin{aligned}
& \widetilde{A_{3}}\left(\left(\xi_{2} \circ \xi_{1}\right)\left(q_{1}\right),\left(\eta_{2} \circ \eta_{1}\right)\left(m_{1}\right),\left(\xi_{2} \circ \xi_{1}\right)\left(p_{1}\right)\right) \\
& =\widetilde{A_{3}}\left(\xi_{2}\left(\xi_{1}\left(q_{1}\right)\right), \eta_{2}\left(\eta_{1}\left(m_{1}\right)\right), \xi_{2}\left(\xi_{1}\left(p_{1}\right)\right)\right) \\
& =\bigvee\left\{\widetilde{A_{2}}\left(\xi_{1}\left(q_{1}\right), \eta_{1}\left(m_{1}\right), r_{1}\right) \mid \xi_{2}\left(r_{1}\right)=\xi_{2}\left(\xi_{1}\left(p_{1}\right)\right)\right\}
\end{aligned}
$$

Since $\xi_{1}$ is onto, so every $r_{2} \in Q_{2}$ can be written as $\xi_{1}\left(s_{1}\right)$ for some $s_{1} \in Q_{1}$. And then, for every $r_{2} \in Q_{2}$ such that $\xi_{2}\left(r_{2}\right)=\xi_{2}\left(\xi_{1}\left(p_{1}\right)\right)$. Thus,

$$
\widetilde{A_{2}}\left(\xi_{1}\left(q_{1}\right), \eta_{1}\left(m_{1}\right), r_{1}\right)=\bigvee\left\{\widetilde{A_{1}}\left(q_{1}, m_{1}, s_{1}\right) \mid \xi_{1}\left(s_{1}\right)=r_{2}\right\}
$$

Hence,

$$
\begin{aligned}
& \widetilde{A_{3}}\left(\left(\xi_{2} \circ \xi_{1}\right)\left(q_{1}\right),\left(\eta_{2} \circ \eta_{1}\right)\left(m_{1}\right),\left(\xi_{2} \circ \xi_{1}\right)\left(p_{1}\right)\right) \\
& =\bigvee\left\{\bigvee\left\{\widetilde{A_{1}}\left(q_{1}, m_{1}, s_{1}\right) \mid \xi_{1}\left(s_{1}\right)=r_{2}\right\} \xi_{2}\left(r_{1}\right)=\left(\xi_{2} \circ \xi_{1}\right)\left(p_{1}\right)\right\} \\
& =\bigvee\left\{\widetilde{A_{1}}\left(q_{1}, m_{1}, s_{1}\right) \mid\left(\xi_{2} \circ \xi_{1}\right)\left(s_{1}\right)=\left(\xi_{2} \circ \xi_{1}\right)\left(p_{1}\right)\right\} .
\end{aligned}
$$


and

$$
\begin{aligned}
& \lambda_{3}\left(\left(\xi_{2} \circ \xi_{1}\right)\left(q_{1}\right),\left(\eta_{2} \circ \eta_{1}\right)\left(m_{1}\right),\left(\xi_{2} \circ \xi_{1}\right)\left(p_{1}\right)\right) \\
& =\lambda_{3}\left(\xi_{2}\left(\xi_{1}\left(q_{1}\right)\right), \eta_{2}\left(\eta_{1}\left(m_{1}\right)\right), \xi_{2}\left(\xi_{1}\left(p_{1}\right)\right)\right) \\
& =\bigwedge\left\{\lambda_{2}\left(\xi_{1}\left(q_{1}\right), \eta_{1}\left(m_{1}\right), r_{1}\right) \mid \xi_{2}\left(r_{1}\right)=\xi_{2}\left(\xi_{1}\left(p_{1}\right)\right)\right\}
\end{aligned}
$$

Since $\xi_{1}$ is onto, so every $r_{2} \in Q_{2}$ can be written as $\xi_{1}\left(s_{1}\right)$ for some $s_{1} \in Q_{1}$. And then, for every $r_{2} \in Q_{2}$ such that $\xi_{2}\left(r_{2}\right)=\xi_{2}\left(\xi_{1}\left(p_{1}\right)\right)$. Thus,

$$
\lambda_{2}\left(\xi_{1}\left(q_{1}\right), \eta_{1}\left(m_{1}\right), r_{1}\right)=\bigwedge\left\{\lambda_{1}\left(q_{1}, m_{1}, s_{1}\right) \mid \xi_{1}\left(s_{1}\right)=r_{2}\right\}
$$

Hence,

$$
\begin{aligned}
& \lambda_{3}\left(\left(\xi_{2} \circ \xi_{1}\right)\left(q_{1}\right),\left(\eta_{2} \circ \eta_{1}\right)\left(m_{1}\right),\left(\xi_{2} \circ \xi_{1}\right)\left(p_{1}\right)\right) \\
& =\bigwedge\left\{\bigwedge\left\{\lambda_{1}\left(q_{1}, m_{1}, s_{1}\right) \mid \xi_{1}\left(s_{1}\right)=r_{2}\right\} \xi_{2}\left(r_{1}\right)=\left(\xi_{2} \circ \xi_{1}\right)\left(p_{1}\right)\right\} \\
& =\bigwedge\left\{\lambda_{1}\left(q_{1}, m_{1}, s_{1}\right) \mid\left(\xi_{2} \circ \xi_{1}\right)\left(s_{1}\right)=\left(\xi_{2} \circ \xi_{1}\right)\left(p_{1}\right)\right\} .
\end{aligned}
$$

(2) Since $\xi_{1}$ and $\xi_{2}$ are one-to-one, then we have, for any $p_{1}, q_{1} \in Q_{1}$ and $m_{1} \in M_{1}$, so

$$
\begin{aligned}
& \widetilde{A_{3}}\left(\left(\xi_{2} \circ \xi_{1}\right)\left(q_{1}\right),\left(\eta_{2} \circ \eta_{1}\right)\left(m_{1}\right),\left(\xi_{2} \circ \xi_{1}\right)\left(p_{1}\right)\right)=\widetilde{A_{2}}\left(\xi_{1}\left(q_{1}\right), \eta_{1}\left(m_{1}\right), \xi_{1}\left(p_{1}\right)\right) \\
& \widetilde{A_{3}}\left(\left(\xi_{2} \circ \xi_{1}\right)\left(q_{1}\right),\left(\eta_{2} \circ \eta_{1}\right)\left(m_{1}\right),\left(\xi_{2} \circ \xi_{1}\right)\left(p_{1}\right)\right)=\widetilde{A_{1}}\left(q_{1}, m_{1}, p_{1}\right)
\end{aligned}
$$

and

$$
\begin{aligned}
& \lambda_{3}\left(\left(\xi_{2} \circ \xi_{1}\right)\left(q_{1}\right),\left(\eta_{2} \circ \eta_{1}\right)\left(m_{1}\right),\left(\xi_{2} \circ \xi_{1}\right)\left(p_{1}\right)\right)=\lambda_{2}\left(\xi_{1}\left(q_{1}\right), \eta_{1}\left(m_{1}\right), \xi_{1}\left(p_{1}\right)\right) \\
& \lambda_{3}\left(\left(\xi_{2} \circ \xi_{1}\right)\left(q_{1}\right),\left(\eta_{2} \circ \eta_{1}\right)\left(m_{1}\right),\left(\xi_{2} \circ \xi_{1}\right)\left(p_{1}\right)\right)=\lambda_{1}\left(q_{1}, m_{1}, p_{1}\right) .
\end{aligned}
$$

Hence, proof.

Definition 7. Let $\aleph=\langle Q, M, \mathscr{A}\rangle$ is a cfsm and take $p, q \in Q$. Then,

(1) $p$ is called a cubic immediate successor of $q$ if there exists $v \in M$ such that $\widetilde{A}(q, v, p) \succ \widetilde{0}$ and $\lambda(q, v, p)<1$

(2) $p$ is called a cubic successor of $q$ and $q$ is called source of $p$ if there exists an element $a \in M^{*}$ such that $\widetilde{A^{*}}(q, a, p) \succ \widetilde{0}$ and $\lambda^{*}(q, a, p)<1$. The set of all cubic successor of $q$ is denoted by $C(q)$ and the set of all cubic sources of $p$ is denoted by $C S(p)$.

Theorem 2. Let $\aleph=\langle Q, M, \mathscr{A}\rangle$ is a cfsm. Then, for any p.q, $r \in Q$, the following properties hold.

(1) $q \in C(q)$

(2) If $p \in C(q)$ and $r \in C(p)$, then $r \in C(q)$.

Proof. 1) Since $\widetilde{A^{*}}(q, v, q)=\widetilde{1} \succ \widetilde{0}$ and $\lambda^{*}(q, v, q)=0<1$, so $q \in C(q)$.

2) Take $p \in C(q)$ and $r \in C(p)$, then there exist $m, a \in M^{*}$ such that $\widetilde{A^{*}}(q, m, p) \succ \widetilde{0}, \lambda^{*}(q, m, p)<1, \widetilde{A^{*}}(p, a, r) \succ \widetilde{0}$ and $\lambda^{*}(p, a, r)<1$. We using Proposition 1, we have

$$
\begin{aligned}
\widetilde{A^{*}}(q, m a, r) & =\bigvee_{s \in Q}\left\{\widetilde{A^{*}}(q, m, s) \wedge \widetilde{A^{*}}(s, a, r)\right\} \\
& \succeq \widetilde{A^{*}}(q, m, p) \wedge \widetilde{A^{*}}(p, a, r) \succ \widetilde{0}
\end{aligned}
$$


and

$$
\begin{aligned}
\lambda^{*}(q, m a, r) & =\bigwedge_{s \in Q}\left\{\lambda^{*}(q, m, s) \vee \lambda^{*}(s, a, r)\right\} \\
& \leq \lambda^{*}(q, m, p) \vee \lambda^{*}(p, a, r)<1 .
\end{aligned}
$$

Hence, $r \in C(q)$.

Definition 8. Let $\boldsymbol{\aleph}=\langle Q, M, \mathscr{A}\rangle$ is a cfsm. Then, the cubic source, cubic successor operator $C_{Q}, C S_{Q}: \wp(Q) \longrightarrow \wp(Q)$ are defined as:

$$
\begin{aligned}
C S_{Q}(T) & =\left\{q \in Q: \widetilde{A^{*}}(q, m, p) \succ \widetilde{0} \text { and } \lambda^{*}(q, m, p)<1 \text { for some }(m, p) \in X^{*} \times T\right\} \\
C_{Q}(T) & =\left\{p \in Q: \widetilde{A^{*}}(q, m, p) \succ \widetilde{0} \text { and } \lambda^{*}(q, m, p)<1 \text { for some }(q, m) \in T \times X^{*}\right\}
\end{aligned}
$$

Note that $C S_{Q}(T)$ and $C_{Q}(T)$ are denoted as $C S(T)$ and $C(T)$, respectively. If no confusion creates, then $C(\{q\})$ and $C S(\{q\})$.

Theorem 3. Let $\aleph=\langle Q, M, \mathscr{A}\rangle$ is a cfsm and let $T$ and $N$ be any subsets of $Q$. Then, the following assertions hold.

(1) If $T \subseteq N$, then $C(T) \subseteq C(N)$ and $C S S(T) \subseteq C S(N)$

(2) $T \subseteq C(T)$ and $T \subseteq C(T)$.

(3) $C(C(T))=C(T)$.

(4) $C(T \cup N)=C(T) \cup C(N)$ and $C S(T \cup N)=C S(T) \cup C(N)$.

(5) $C(T \cap N) \subseteq C(T) \cap C(N)$.

(6) $C(T)=T$ if and only if $C S(Q \backslash T)=Q \backslash T$

Proof. The proofs of (1), (2), (4), (5) are straightforward. We only prove (3), obviously $C(T) \subseteq C(C(T))$. Let $q \in C(C(T))$. Then, $q \in C(p)$ for some $p \in C(T)$. If $p \in C(T)$, then there exist $s \in T$ such that $p \in C(s)$. Using Theorem 2, we have $q \in C(s) \subseteq C(T)$ so that $C(C(T)) \subseteq C(T)$. Hence, (3) is true.

(6) Assume $C(T)=T$ and $q \in C S(Q \backslash T)$. Then, $q \in C S(p)$ for some $p \in Q \backslash T$. Thus, $p \in C(T)$. If $q \in T$, then $p \in C(q) \subseteq C(T)=T$, which is a contradiction with the fact $p \in Q \backslash T$. Hence, $q \in Q \backslash T$ and $C S(Q \backslash T) \subseteq Q \backslash T$. Since $Q \backslash T \subseteq C S(Q \backslash T)$, this follows that $C S(Q \backslash T)=Q \backslash T$.

Conversely, suppose $C S(Q \backslash T)=Q \backslash T$. Let $q \in C(T)$. Then, $q \in C(p)$ for some $p \in T$. Thus, $p \in C S(q)$. If $q \in Q \backslash T$, then $p \in C S(q) \subseteq C S(Q \backslash T)=Q \backslash T$, which contradicts with the assumption of $p \in T$. Thus, $q \in T$. So, $C(T) \subseteq T$. Since $T \subseteq C(T)$, it follows that $C(T)=T$.

Definition 9. Let $\mathbb{\aleph}=\langle Q, M, \mathscr{A}\rangle$ is a cfsm. Then, satisfies the cubic exchange property if for all $p, q \in Q$ and $T$ is any subset of $Q$ such that $p \in C(T \cup\{q\})$ and $p \notin C(T)$, then $q \in C(T \cup\{p\})$.

Theorem 4. Let $\boldsymbol{\aleph}=\langle Q, M, \mathscr{A}\rangle$ is a cfsm. Then, the following axioms are equivalent.

(1) holds the cubic exchange property.

(2) $(\forall p, q \in Q)(p \in C(q) \Leftrightarrow q \in C(p))$.

Proof. Suppose that $\boldsymbol{\aleph}$ holds the cubic exchange property. Take $p, q$ in $Q$ such that $p \in C(q)=C(\emptyset \cup\{q\})$. Since $p \notin C(\emptyset)$ so $q \in C(\emptyset \cup\{p\})=C(p)$. Similarly, we prove that if $q \in C(p)$, then $p \in C(q)$. 
Conversely, suppose that (2) is true. Take $p, q$ in $Q$ and $T$ is a subset of $Q$. If $p \in C(T \cup\{q\})$ and $p \notin C(T)$, then by using (2), we have

$$
q \in C(p) \subseteq C(T \cup\{p\})
$$

Hence, $\boldsymbol{\aleph}$ holds the cubic exchange property.

Definition 10. Let $\mathfrak{\aleph}=\langle Q, M, \mathscr{A}\rangle$ be a cfsm and let $\mathscr{B}=\langle\widetilde{B}, \mu\rangle$ be a cubic set in $Q$. Then, $\langle Q, \mathscr{B}, M, \mathscr{A}\rangle$ is said to be a cubic subsystem of $\aleph$ if for all $p, q \in Q$ and $m \in M$, the following conditions hold.

$$
\widetilde{B}(q) \succeq \operatorname{rinf}\{\widetilde{B}(p), \widetilde{B}(q, m, p)\} \text { and } \mu(q) \leq r \inf \{\mu(p), \mu(q, m, p)\}
$$

If $\langle Q, \mathscr{B}, M, \mathscr{A}\rangle$ is a cubic subsystem, we will write $\mathscr{B}$, instead of $\langle Q, \mathscr{B}, M, \mathscr{A}\rangle$.

Example 2. Let $Q=\{p, q\}, M=\{m\}, \widetilde{A}(q, m, p)=\widetilde{A}(p, m, q)=[0.45,0.55]$ and $\lambda(q, m, p)=\lambda(p, m, q)=0.5$. Now define $\mathscr{B}=\langle\widetilde{B}, \mu\rangle$ in $Q$ as $\widetilde{B}(q)=[0.7,0.8], \widetilde{B}(p)=[0.45,0.55], \mu(q)=0.7$ and $\mu(q)=0.5$. Then, clearly $\mathscr{B}$ is a cubic subsystem.

Example 3. Let $Q=\{p, q, r\}, M=\left\{m_{1}, m_{2}\right\}, \widetilde{A}\left(t, m_{1}, s\right)=[0.45,0.5], \widetilde{A}(t, m, s)=[0.55,0.6]$ and $\lambda\left(t, m_{1}, s\right)=0.55$, $\lambda(t, m, s)=0.6$ for all $t, s \in Q$. Let $\mathscr{B}=\langle\widetilde{B}, \mu\rangle$ be given by $\widetilde{B}(q)=[0.7,0.75], \widetilde{B}(p)=[0.78,0.8], \widetilde{B}(p)=[0.55,0.6]$ and $\mu(q)=0.7, \mu(p)=0.75, \mu(p)=0.6$. Hence, by routine calculation $\mathscr{B}$ is a cubic subsystem.

Theorem 5. Let $\aleph=\langle Q, M, \mathscr{A}\rangle$ be a cfsm and let $\mathscr{B}=\langle\widetilde{B}, \mu\rangle$ be a cubic set in $Q$. Then, $\mathscr{B}$ is a cubic subsystem $\aleph$ if and only if

$$
\widetilde{B}(q) \succeq \widetilde{B}(p) \wedge \widetilde{A^{*}}(q, m, p) \text { and } \mu(q) \leq\left\{\mu(p) \wedge \lambda^{*}(q, m, p)\right\}
$$

for all $p . q \in Q$ and $m \in M^{*}$.

Proof. Assume that $\mathscr{B}$ is a cubic subsystem of and let $p . q \in Q$ and $m \in M^{*}$. We will use the induction for proof $|m|=n$. In case of $n=0$, then $m=\varepsilon$. Now if $p=q$, then

$$
\widetilde{B}(p) \wedge \widetilde{A^{*}}(q, m, p)=\widetilde{B}(q) \text { and }\left\{\mu(p) \wedge \lambda^{*}(q, m, p)\right\}=\mu(p) .
$$

If $p \neq q$

$$
\widetilde{B}(p) \wedge \widetilde{A^{*}}(q, m, p)=\widetilde{0} \preceq \widetilde{B}(q) \text { and }\left\{\mu(p) \wedge \lambda^{*}(q, m, p)\right\}=0 \leq \mu(q) .
$$

Thus, the result is valid for $n=0$. Now assume the result is true for all $y \in M^{*}$ such that $|y|=n-1, n>0$. Let $m=y x$, where $x \in M$. Then,

$$
\begin{aligned}
\widetilde{B}(p) \wedge \widetilde{A^{*}}(q, m, p) & =\widetilde{B}(p) \wedge \widetilde{A^{*}}(q, y x, p) \\
& =\widetilde{B}(p) \wedge\left(\bigvee_{s \in Q}\left\{\widetilde{A^{*}}(q, y, s) \wedge \widetilde{A}(s, x, p)\right\}\right) \\
& =\bigvee_{s \in Q}\left\{\widetilde{B}(p) \wedge \widetilde{A^{*}}(q, y, s) \wedge \widetilde{A}(s, x, p)\right\} \\
& =\bigvee_{s \in Q}\{\widetilde{B}(p) \wedge \widetilde{A}(s, x, p)\} \preceq \widetilde{B}(q)
\end{aligned}
$$


and

$$
\begin{aligned}
\mu(p) \vee \lambda^{*}(q, m, p) & =\mu(p) \vee \lambda^{*}(q, y x, p) \\
& =\mu(p) \vee\left(\bigwedge_{s \in Q}\left\{\lambda^{*}(q, y, s) \vee \lambda(s, x, p)\right\}\right) \\
& =\bigwedge_{s \in Q}\left\{\mu(p) \vee \lambda^{*}(q, y, s) \vee \lambda(s, x, p)\right\} \\
& =\bigwedge_{s \in Q}\{\mu(p) \vee \lambda(s, x, p)\} \geq \lambda(q) .
\end{aligned}
$$

Conversely, straightforward.

Definition 11. A cfsm $\aleph=\langle Q, M, \mathscr{A}\rangle$ is called a cubic retrievable if

$$
\begin{aligned}
& (\forall q \in Q)\left(\forall m \in M^{*}\right)(\exists t \in Q)\left(\widetilde{A^{*}}(q, m, t) \succ \widetilde{0} \text { and } \lambda^{*}(q, m, t)<1\right) \\
& \Rightarrow\left(\exists a \in M^{*}\right)\left(\widetilde{A^{*}}(t, a, q) \succ \widetilde{0} \text { and } \lambda^{*}(t, a, q)<1\right) .
\end{aligned}
$$

Definition 12. Let $\mathfrak{\aleph}=\langle Q, M, \mathscr{A}\rangle$ be a cfsm and take $q, r, t \in Q$. Then, $r$ and $t$ are called cubic $q$-related if there exists $m \in M^{*}$ such that $\widetilde{A^{*}}(q, m, r) \succ \widetilde{0}, \widetilde{A^{*}}(q, m, t) \succ \widetilde{0}, \lambda^{*}(q, m, r)<1$ and $\lambda^{*}(q, m, t)<1$.

We say that $r$ and $t$ are cubic $q$-twins if

(i) $r$ and t are called cubic $q$-related,

(ii) $C(r)=C(t)$.

Proposition 2. Let $=\langle Q, M, \mathscr{A}\rangle$ be a cfsm. Then, the following conditions are equivalent.

(1) $\forall q, r, t \in Q$, if $r$ and $t$ are cubic $q$-related, then $r$ and $t$ are cubic $q$-twins.

(2) $(\forall q, r, t \in Q)\left(\forall a, m \in M^{*}\right) \widetilde{A^{*}}(q, m, r) \succ \widetilde{0}, \widetilde{A^{*}}(q, m, t) \succ \widetilde{0}, \lambda^{*}(q, m, r)<1$ and $\left.\lambda^{*}(q, m, t)<1 \Rightarrow p \in C(r)\right)$.

Proof. (1) $\Rightarrow(2)$ : Take $p, q, r \in Q$ and $a, m \in M^{*}$ be such that

$$
\widetilde{A^{*}}(q, m, r) \succ \widetilde{0}, \widetilde{A^{*}}(q, m a, p) \succ \widetilde{0}, \lambda^{*}(q, m, r)<1 \text { and } \lambda^{*}(q, m a, p)<1 .
$$

Since,

$$
\widetilde{A^{*}}(q, m a, p)=\bigvee_{t \in Q}\left\{\widetilde{A^{*}}(q, m, t) \wedge \widetilde{A^{*}}(t, a, p)\right\} \succ \widetilde{0}
$$

and

$$
\lambda^{*}(q, m a, p)=\bigwedge_{t \in Q}\left\{\lambda^{*}(q, m, t) \vee \lambda^{*}(t, a, p)\right\}<1
$$

Then, by Proposition 1, there exists $t \in Q$ such that $\widetilde{A^{*}}(q, m, t) \succ \widetilde{0}, \widetilde{A^{*}}(t, a, p) \succ \widetilde{0}, \lambda^{*}(q, m, t)<1, \lambda^{*}(t, a, p)<1$. This mean that $r$ and $t$ are cubic $q$-related. This follows from (1), $r$ and $t$ are cubic $q$-twins, so that $p \in C(r)=C(t)$.

$(2) \Rightarrow(1):$ Assume that (2) is true. Take $p, q, r \in Q$ be such that $r$ and $t$ are cubic $q$-related. Then, there exists $m \in M^{*}$ such that $\widetilde{A^{*}}(q, m, t) \succ \widetilde{0}, \widetilde{A^{*}}(t, m, p) \succ \widetilde{0}, \lambda^{*}(q, m, t)<1, \lambda^{*}(t, m, p)<1$. If $p \in C(t)$, then there exists $a \in M^{*}$ such that $\widetilde{A^{*}}(q, a, t) \succ \widetilde{0}, \widetilde{A^{*}}(t, a, p) \succ \widetilde{0}, \lambda^{*}(q, a, t)<1, \lambda^{*}(t, a, p)<1$. Thus,

$$
\widetilde{A^{*}}(q, m a, p)=\bigvee_{t \in Q}\left\{\widetilde{A^{*}}(q, m, t) \wedge \widetilde{A^{*}}(t, a, p)\right\} \succ \widetilde{0}
$$


and

$$
\lambda^{*}(q, m a, p)=\bigwedge_{t \in Q}\left\{\lambda^{*}(q, m, t) \vee \lambda^{*}(t, a, r)\right\}<1 .
$$

Hence, $p \in C(r)$ by given condition. Similarly, if $p \in C(t)$, then $p \in C(r)$. Thus, $r$ and $t$ are cubic $q$-twins.

Theorem 6. Let $\mathfrak{\aleph}=\langle Q, M, \mathscr{A}\rangle$ be a cfsm. Then, $\mathfrak{\aleph}$ is a cubic retrievable if and only if it hold

(1)

$$
\begin{aligned}
& (\forall q \in Q)\left(\forall m \in M^{*}\right)(\exists t \in Q)\left(\widetilde{A^{*}}(q, m, t) \succeq \widetilde{0} \text { and } \lambda^{*}(q, m, t)<1\right) \\
& \Rightarrow\left(\exists a \in M^{*}\right)\left(\widetilde{A^{*}}(q, m a, q) \succeq \widetilde{0} \text { and } \lambda^{*}(q, m a, q)<1\right) .
\end{aligned}
$$

(2) $\forall q, r, t \in Q$, if $r$ and t are cubic $q$-related, then $r$ and t are cubic $q$-twins.

Proof. Straightforward

\section{Cubic transformation semigroups}

In this section, we define cubic transformation semigroups and related properties. The given concept is a generalization of fuzzy transformation semigroup and intuitionistic fuzzy transformation semigroups.

Definition 13. A cubic transformation semigroup (in short, cts) is a triplet $\mathscr{S}=\langle Q, X, \mathscr{A}\rangle$, where $Q$ is a non-empty finite set, $X$ is a finite semigroup and $\mathscr{A}=\langle\widetilde{A}, \lambda\rangle$ is a cubic set in $Q \times X \times Q$ such that (TS1)

$$
\widetilde{A}(q, \varepsilon, p)=\left\{\begin{array}{l}
\widetilde{1} \text { if } q=p \\
\widetilde{0} \text { if } q \neq p
\end{array} \quad \lambda(q, \varepsilon, p)=\left\{\begin{array}{l}
0 \text { if } q=p \\
1 \text { if } q \neq p
\end{array}\right.\right.
$$

$(T S 2)$

$$
\begin{aligned}
& \widetilde{A}(q, x y, p)=\bigvee_{r \in Q}\{\widetilde{A}(q, x, r) \wedge \widetilde{A}(r, y, p)\} \\
& \lambda(q, x y, p)=\bigwedge_{r \in Q}\{\lambda(q, x, r) \vee \lambda(r, y, p)\}
\end{aligned}
$$

for all $p, q \in Q, x, y \in M$.

If addition, if $\mathscr{S}=\langle Q, X, \mathscr{A}\rangle$ holds the property $\left(\begin{array}{l}\widetilde{A}(q, x, p)=\widetilde{A}(q, y, p) \\ \lambda(q, x, p)=\lambda(q, y, p)\end{array}\right) \Rightarrow x=y$ for any $x, y \in X$ and $p, q \in Q$. Then, $\mathscr{S}=\langle Q, X, \mathscr{A}\rangle$ is called a faithful cubic transformation semigroup.

Now take $M^{*}$ is free monoid with respect to the binary operation concatenation of two words. Let $m, a \in M^{*}$. Define a relation $\sim$ on $M^{*}$ by $m \sim a \Leftrightarrow \widetilde{A^{*}}(q, m, p)=\widetilde{A^{*}}(q, a, p)$ and $\lambda^{*}(q, m, p)=\lambda^{*}(q, a, p)$ for all $p, q \in Q$.

Lemma 1. Let $\boldsymbol{\aleph}=\langle Q, M, \mathscr{A}\rangle$ be a cfsm. Then, a relation $\sim$ on $M^{*}$ is an equivalence relation, where $M^{*}$ is a semigroup with identity $\varepsilon$.

Proof. Let $p, q \in Q$ and $m, a \in M^{*}$. Then, clearly $\sim$ is reflexive. i.e $\widetilde{A^{*}}(q, m, p)=\widetilde{A^{*}}(q, m, p)$ and $\lambda^{*}(q, m, p)=\lambda^{*}(q, m, p)$. Thus, $m \sim a$. 
Symmetric: Now let $m \sim a$. Then,

$\widetilde{A^{*}}(q, m, p)=\widetilde{A^{*}}(q, a, p)$ and $\lambda^{*}(q, m, p)=\lambda^{*}(q, a, p)$

$\widetilde{A^{*}}(q, a, p)=\widetilde{A^{*}}(q, m, p)$ and $\lambda^{*}(q, a, p)=\lambda^{*}(q, m, p)$.

Hence, $a \sim m$.

Transitive: Take $p, q \in Q$ and $m, a, b \in M^{*}$ be such that $m \sim a$ and $a \sim b$. Then,

$$
\widetilde{A^{*}}(q, m, p)=\widetilde{A^{*}}(q, a, p) \text { and } \lambda^{*}(q, m, p)=\lambda^{*}(q, a, p)
$$

and

$$
\widetilde{A^{*}}(q, a, p)=\widetilde{A^{*}}(q, b, p) \text { and } \lambda^{*}(q, a, p)=\lambda^{*}(q, b, p) .
$$

From equation 1 and 2, its follow that

$$
\begin{aligned}
& \widetilde{A^{*}}(q, m, p)=\widetilde{A^{*}}(q, a, p)=\widetilde{A^{*}}(q, b, p) \text { and } \lambda^{*}(q, m, p)=\lambda^{*}(q, a, p)=\lambda^{*}(q, b, p) \\
& \widetilde{A^{*}}(q, m, p)=\widetilde{A^{*}}(q, b, p) \text { and } \lambda^{*}(q, m, p)=\lambda^{*}(q, b, p) \Rightarrow m \sim b .
\end{aligned}
$$

Hence, $\sim$ is an equivalence relation on $M^{*}$.

For any $m \in M^{*}$, we denote $[m]=\left\{a \in M^{*} \mid m \sim a\right\}$ and $E(\boldsymbol{\aleph})=\left\{[m] \mid m \in M^{*}\right\}$.

Proposition 3. Let $\boldsymbol{\aleph}=\langle Q, M, \mathscr{A}\rangle$ be a cfsm and define a binary operation $\odot$ on $E(\aleph)$ by $[\mathrm{m}] \odot[a]=[\mathrm{ma}]$ for all $[m] \odot[a] \in E(\aleph)$. Then $(E(\aleph), \odot)$ is a semigroup.

Proof. Straightforward.

Example 4. Let $\boldsymbol{\aleph}=\langle Q, M, \mathscr{A}\rangle$ be cfsm, where $Q=\{q\}$ and $X=\left\{x_{1}, x_{2}\right\}$ and $\mathscr{A}=\langle\widetilde{A}, \lambda\rangle$ is defined by

$$
\begin{aligned}
& \widetilde{A}\left(q, x_{1}, q\right)=\widetilde{A}\left(q, x_{1}, q\right)=[0.5,0.6] \\
& \lambda\left(q, x_{1}, q\right)=\lambda\left(q, x_{1}, q\right)=0.5 .
\end{aligned}
$$

For any $a, b \in M^{*}$, we have

$$
\begin{aligned}
\widetilde{A^{*}}(q, a, q) & =\widetilde{A^{*}}(q, b, q)=[0.5,0.55] \\
\lambda(q, a, q) & =\lambda(q, b, q)=0.6
\end{aligned}
$$

Hence, $a \sim b$ and so $E(\aleph)=\{[\varepsilon],[a]\}$, obviously $[\varepsilon]$ is identity and $[a] \odot[a]=[a]$.

We can easily construct a faithful transformation semigroup from any transformation semigroup in the case of cubic set. Define a relation $\approx$ on $X$ by $x \approx y \Leftrightarrow(p, q \in Q)\left(\begin{array}{c}\widetilde{A}(q, x, p)=\widetilde{A}(q, y, p) \\ \lambda(q, x, p)=\lambda(q, y, p)\end{array}\right)$ for all $x, y \in X$. Hence " $\approx$ " is a equivalence relation on $X$. Let $[[x]]$ denote the equivalence class of $\approx$ induced by $x$. Let $X / \approx=\{[[x]]: x \in X\}$.

Theorem 7. 'Let $\mathscr{S}=\langle Q, X, \mathscr{A}\rangle$ be cft and $\approx$ a equivalence relation on $X$. Then,

(i) The equivalence relation $\approx$ is a congruence and the quotient set $X / \approx$ is finite semigroup. 
(ii) The triple $\mathscr{S}=\langle Q, X / \approx, \overline{\mathscr{A}}\rangle$ is a faithful cubic transformation semigroup, where $\bar{A}: Q \times X / \approx \times Q \longrightarrow[I] \times[0,1]$ is given by

$$
\widetilde{\bar{A}}(q,[[x]], p)=\widetilde{A}(q, x, p) \text { and } \bar{\lambda}(q,[[x]], p)=\lambda(q, x, p)
$$

for all $[[x]] \in X / \approx$ and $p, q \in Q$.

Proof.

(i) Take $x, y \in X$ such that $x \approx y$. If $w \in X$, then, for any $p, q \in Q$.

$$
\begin{aligned}
\widetilde{A}(q, x w, p) & =\bigvee_{r \in Q}\{\widetilde{A}(q, x, r) \wedge \widetilde{A}(r, w, p)\} \\
& =\bigvee_{r \in Q}\{\widetilde{A}(q, y, r) \wedge \widetilde{A}(r, w, p)\} \\
& =\widetilde{A}(q, y w, p)
\end{aligned}
$$

and

$$
\begin{aligned}
\lambda(q, x w, p) & =\bigwedge_{r \in Q}\{\lambda(q, x, r) \vee \lambda(r, w, p)\} \\
& =\bigwedge_{r \in Q}\{\lambda(q, y, r) \vee \lambda(r, w, p)\} \\
& =\lambda(q, y w, p) .
\end{aligned}
$$

So, $x w \approx y w$. Similarly we can prove if $x \approx w \Rightarrow y x \approx y w$. Hence, $\approx$ is a congruence. Therefore $X / \approx$ is finite semigroup with binary operation defined by $[[x]] *[[x]]=[[x y]]$.

(ii) The mapping $\overline{\mathscr{A}}: Q \times X / \approx \times Q \longrightarrow[I] \times[0,1]$ is well defined. Next we prove that $\mathscr{S}=\langle Q, X / \approx, \overline{\mathscr{A}}\rangle \mathrm{cft}$. If $X$ is a finite semigroup with identity $e$, then $[[e]]$ is the identity of $X / \approx$. Since

$$
\begin{aligned}
& \widetilde{\bar{A}}(q,[[e]], p)=\widetilde{A}(q, e, p)=\left\{\begin{array}{l}
\tilde{1} \text { if } q=p, \\
\widetilde{0} \text { if } q \neq p,
\end{array}\right. \\
& \bar{\lambda}(q,[[e]], p)=\lambda(q, e, p)=\left\{\begin{array}{l}
0 \text { if } q=p, \\
1 \text { if } q \neq p .
\end{array}\right.
\end{aligned}
$$

Thus, we conclude that $T S 1$ holds.

If we consider any $x, w \in X$, and any $p, q \in Q$, then

$$
\begin{aligned}
\widetilde{\bar{A}}(q,[[x]][[w]], p) & =\widetilde{A}(q, x w, p)=\bigvee_{r \in Q}\{\widetilde{A}(q, x, r) \wedge \widetilde{A}(r, w, p)\} \\
& =\bigvee_{r \in Q}\{\widetilde{\bar{A}}(q,[[x]], r) \wedge \widetilde{\bar{A}}(r,[[w]], p)\}
\end{aligned}
$$

and

$$
\begin{aligned}
\bar{\lambda}(q,[[x]][[w]], p) & =\lambda(q, x w, p)=\bigwedge_{r \in Q}\{\lambda(q, x, r) \vee \lambda(r, w, p)\} \\
& =\bigwedge_{r \in Q}\{\bar{\lambda}(q,[[x]], r) \vee \bar{\lambda}(r,[[w]], p)\}
\end{aligned}
$$


Thus, TS2 holds.

Finally, for any $[[x]],[[y]] \in X / \approx$ and for any $p, q \in Q$

$$
\begin{aligned}
& \widetilde{\bar{A}}(q,[[x]], p)=\widetilde{\bar{A}}(q,[[y]], p) \Rightarrow \widetilde{A}(q, x, p)=\widetilde{A}(q, w, p) \Rightarrow x=y \\
& \widetilde{\bar{A}}(q,[[x]], p)=\widetilde{\bar{A}}(q,[[y]], p) \Rightarrow[[x]]=[[y]] .
\end{aligned}
$$

Hence, $\overline{\mathscr{S}}=\langle Q, X / \approx, \overline{\mathscr{A}}\rangle$ is a faithful.

Definition 14. Let $\mathscr{S}_{1}=\left\langle Q_{1}, X_{1}, \overline{\mathscr{A}}_{1}\right\rangle$ and $\mathscr{S}_{2}=\left\langle Q_{2}, X_{2}, \overline{\mathscr{A}}_{2}\right\rangle$ be ctss. A cts homomorphism from $\mathscr{S}_{1}$ to $\mathscr{S}_{2}$ is a fair $(\xi, \eta)$ such that

(i) $\xi: Q_{1} \longrightarrow Q_{2}$ is a map.

(ii) $\eta: X_{1} \longrightarrow X_{2}$ is a semigroup homomorphism.

(iii) If $X_{1}$ and $X_{2}$ are semigroup having identity elements $e_{1} \in X_{1}$ and $e_{2} \in X_{2}$, then $\eta\left(e_{1}\right)=e_{2}$.

(iv) For any $p_{1}, q_{1} \in Q_{1}$ and $x_{1} \in X_{1}$.

$$
\widetilde{A_{1}}\left(q_{1}, x_{1}, p_{1}\right) \preceq \widetilde{A_{2}}\left(\xi\left(q_{1}\right), \eta\left(x_{1}\right), \xi\left(p_{1}\right)\right)
$$

and

$$
\lambda_{1}\left(q_{1}, x_{1}, p_{1}\right) \geq \lambda_{2}\left(\xi\left(q_{1}\right), \eta\left(x_{1}\right), \xi\left(p_{1}\right)\right) .
$$

We denoted by $(\xi, \eta): \mathscr{S}_{1} \longrightarrow \mathscr{S}_{2}$.

A cts homomorphism $(\xi, \eta)$ is called strong if, in addition, for any $p_{1}, q_{1} \in Q_{1}$ and $x_{1} \in X_{1}$,

$$
\widetilde{A_{2}}\left(\xi\left(q_{1}\right), \eta\left(x_{1}\right), \xi\left(p_{1}\right)\right)=\bigvee\left\{\widetilde{A_{1}}\left(q_{1}, x_{1}, r_{1}\right) \mid \xi\left(r_{1}\right)=\xi\left(p_{1}\right)\right\}
$$

and

$$
\lambda_{2}\left(\xi\left(q_{1}\right), \eta\left(x_{1}\right), \xi\left(p_{1}\right)\right)=\bigwedge\left\{\lambda_{1}\left(q_{1}, x_{1}, r_{1}\right) \mid \eta\left(r_{1}\right)=\eta\left(p_{1}\right)\right\}
$$

A cts homomorphism $(\xi, \eta)$ is called isomorphism if, both mappings $\xi$ and $\eta$ are bijective.

Remark. If $(\xi, \eta): \aleph_{1} \longrightarrow \aleph_{2}$ is a strong cts homomorphism and $\xi$ one-to-one, then for any $p_{1}, q_{1} \in Q_{1}$ and $x_{1} \in X_{1}$

$$
\widetilde{A}\left(q_{1}, m_{1}, r_{1}\right)=\widetilde{A_{2}}\left(\left(\xi\left(q_{1}\right), \eta\left(m_{1}\right), \xi\left(p_{1}\right)\right)\right.
$$

and

$$
\lambda_{1}\left(q_{1}, m_{1}, r_{1}\right)=\lambda_{2}\left(\xi\left(q_{1}\right), \eta\left(m_{1}\right), \xi\left(p_{1}\right)\right)
$$

Theorem 8. The set of all cfts together with all the cts homomorphism constitutes a category.

Proof. First, since the fair $\left(i d_{Q}, i d_{X}\right)$ is an cts homomorphism for any cts $\mathscr{S}=\langle Q, X / \approx, \overline{\mathscr{A}}\rangle$. Next we prove, if $\mathscr{S}_{1}, \mathscr{S}_{2}$, $\mathscr{S}_{3}$ are cubic transformation semigroups and let us consider the cts homomorphisms $\left(\xi_{1}, \eta_{1}\right): \mathscr{S}_{1} \longrightarrow \mathscr{S}_{2}$ and $\left(\xi_{2}, \eta_{2}\right)$ : $\mathscr{S}_{2} \longrightarrow \mathscr{S}_{3}$. Then, the composition $\left(\xi_{2} \circ \xi_{1}, \eta_{1} \circ \eta_{2}\right): \mathscr{S}_{1} \longrightarrow \mathscr{S}_{3}$ is also homomorphism, denoted by $\left(\xi_{2}, \eta \xi_{12}\right) \circ\left(\xi_{1}, \eta_{1}\right)$.

The next result, as well as his proof, is same as Theorem 1.

Theorem 9. Let $\mathscr{S}_{1}, \mathscr{S}_{2}, \mathscr{S}_{3}$ be ctss and let $\left(\xi_{1}, \eta_{1}\right): \mathscr{S}_{1} \longrightarrow \mathscr{S}_{2}$ and $\left(\xi_{2}, \eta_{2}\right): \mathscr{S}_{2} \longrightarrow \mathscr{S}_{3}$ be both strong cts homomorphisms.

(i) If $\xi_{1}$ is onto, then $\left(\xi_{2}, \eta_{2}\right) \circ\left(\xi_{1}, \eta_{1}\right): \mathscr{S}_{1} \longrightarrow \mathscr{S}_{3}$ is a strong cts homomorphism.

(ii) If $\xi_{1}, \xi_{2}$ are one to one, then the composed cts homomorphism is also strong. 


\section{Competing interests}

The authors declare that they have no competing interests.

\section{Authors' contributions}

All authors have contributed to all parts of the article. All authors read and approved the final manuscript.

\section{References}

[1] Y.H. Kim, J.G. Kim, S.J. Cho, Products of T-generalized state machines and T-generalized transformation semigroups, Fuzzy Sets and Systems 93 (1998) 87-97.

[2] D.S. Malik, J.N. Mordeson, M.K. Sen, On subsystems of a fuzzy finite state machine, Fuzzy Sets and Systems 68 (1994) 83-92.

[3] D.S. Malik, J.N. Mordeson, M.K. Sen, Products of fuzzy finite state machines, Fuzzy Sets and Systems 92 (1997) 95-102.

[4] D.S. Malik, J.N. Mordeson, M.K. Sen, Semigroups of fuzzy finite state machines, in: P.P. Wang (Ed.), Advances in Fuzzy Theory and Technology, Vol. II, 1994, pp. 87-98.

[5] D.S. Malik, J.N. Mordeson, M.K. Sen, Submachines of fuzzy finite state machines, J. Fuzzy Math. 4 (1994) 781-792

[6] H. Bustince, E. Barrenechea, M. Pagola, J. Fernndez, Interval-valued fuzzy sets constructed from matrices: application to edge detection, Fuzzy Sets Syst. 160, (2009), 1819-1840.

[7] R. Belohlavek, Determinism and fuzzy automata, Inform. Sci. 143 (1) (2002) 205-209.

[8] J. Ignjatovic, M. Ciric, S. Bogdanovic', Determinations of fuzzy automata with membership values in complete residuated lattices, Inform. Sci. 178, (2008) 164-180.

[9] H.X. Lei, Y.M. Li, Minimization of states in automata theory based on finite lattice-ordered monoids, Inform. Sci. 177, (6) (2007), 1413-1421.

[10] Y.M. Li, W. Pedrycz, Minimization of lattice finite automata and its application to the decomposition of lattice languages, Fuzzy Sets Syst. 158, (2007), 1423-1436.

[11] Ping Li, Y.M. Li, Algebraic properties of LA-languages, Inform. Sci. 176 (21) (2006) 3232-3255.

[12] H.Z. Li, P. Li, Y.Y. Li, The relationships among several types of fuzzy automata, Inform. Sci. 176 (15) (2006) $2208-2226$.

[13] K. Peeva, Zl. Zahariev, Computing behavior of finite fuzzy machines - algorithm and its application to reduction and minimization, Inform. Sci. 178 (21), (2008), 4152-4165.

[14] D.W. Qiu, Notes on automata theory based on quantum logic, Science in China Series F: Inform. Sci. 50 (2) (2007) 154-169.

[15] S.P. Tiwari, Arun K. Srivastava, On a decomposition of fuzzy automata, Fuzzy Sets Syst. 151 (2005) 503-511.

[16] Y. B. Jun, Intuitionistic fuzzy transformation semigroups, Inform. Sci. 179 (24) (2009) 4284-4291.

[17] Y.B. Jun, Intuitionistic fuzzy finite state machines, J. Appl. Math. Comput. 17 (1-2) (2005) 109-120.

[18] Y.B. Jun, Intuitionistic fuzzy finite switchboard state machines, J. Appl. Math. Comput. 20 (1-2) (2006) 315-325.

[19] Y.B. Jun, Quotient structures of intuitionistic fuzzy finite state machines, Inform. Sci. 177 (2007) 4977-4986

[20] W.G. Wee, On generalization of adaptive algorithm and application of fuzzy sets concept to pattern classification. PH.D Theses, Purdue University, June, 1967.

[21] E. Orlowska, Semantic analysis of inductive reasoning, Theoret. Comput. Sci. 43 (1986) 81-89.

[22] A. Kandal, Fuzzy Switching and Automata: Theory and Applications (Crane Russak, 1980).

[23] W. M. Holcombe, Algebraic Automata Theory (Cambridge Univ. Press, Cambridge, 1982).

[24] L. A. Zadeh, Fuzzy sets, Inform. Control 8, (1965), 338-353.

[25] H. V. Kumbhojkar and S. R. Chaudhari, On covering of products of fuzzy finite state machines, Fuzzy Sets and Systems 125 (2002), 215-222.

[26] K. Peeva, Zl. Zahariev, Computing behavior of finite fuzzy machines - algorithm and its application to reduction and minimization, Inform. Sci. 178 (21), (2008) 4152-4165.

[27] D.W. Qiu, Notes on automata theory based on quantum logic, Science in China Series F: Inform. Sci. 50 (2), (2007), 154-169. 
[28] D.S. Malik, J.N. Mordeson, Structure of upper and lower approximation spaces of infinite sets, in: T.Y. Lin, Y.Y. Yao, L.A. Zadeh (Eds.), Data Mining,

[29] Rough Sets and Granular Computing, Studies in Fuzziness and Soft Computing, vol. 95, Physica-Verlag, Heidelberg, New York, 2002, pp. 461-472.

[30] Z. Pawlak, Rough Sets, Theoretical Aspects about Data, Kluwer Academic Publisher, Dordrecht, 1991.

[31] N. Kuroki, J.N. Mordeson, Successor and source functions, J. Fuzzy Math. 5 (1997) 173-182

[32] N. Kuroki, J.N. Mordeson, Successor and source functions, J. Fuzzy Math. 5 (1997) 173-182

[33] Z. Bavel, Introduction to the Theory of Automata, Reston Publishing Company, Inc., Reston, Virginia, 1983.

[34] E.S. Santos, On reduction of max-min machines, J. Math. Anal. Appl. 37 (1972) 677-686.

[35] E.S. Santos, Fuzzy automata and languages, Inform. Sci. 10 (1976) 193-197.

[36] Y. B. Jun, C. S. Kim, K. O. Yang, Cubic sets, Annals of fuzzy Mathematics and Informatics, 4(2012), 83-98.

[37] S. Eilenberg, "Automata, Languages, and Machines," Academic Press, New York, vol. A, B, 1974.

[38] S. Kleene, "Representation of events in nerve nets and finite automata," in: C.E. Shannon and J. McCarthy (eds.), Automata Studies, Princeton University Press, pp. 3-42, 1956.

[39] S. Yu, "Regular languages," in: G. Rozenberg, A. Salomaa (Eds.), Handbook of Formal Languages, Springer-Verlag, Berlin, Heidelberg, vol. 1, pp. 41-110, 1997.

[40] S.P. Tiwari, Arun K. Srivastava, On a decomposition of fuzzy automata, Fuzzy Sets Syst. 151 (2005) 503-511

[41] A. V. Aho and J. D. Ullman , "Foundations of Computer Science," Computer Science Press, New York, 1994.

[42] L. A. Zadeh, Fuzzy Sets, Inform. \& Control 8, (1965), 338 -353. 